[0212-7199 (2005) 22: 5; pp 209-212] ANALES DE MEDICINA INTERNA Copyright $(2005$ ARAN EDICIONES, S.L.

AN. MED. INTERNA (Madrid) Vol. 22, N. ${ }^{\circ}$ 5, pp. 209-212, 2005

\title{
Alteraciones gasométricas en pacientes cirróticos hospitalizados
}

\author{
V. LORENZO-ZÚÑIGA, M. A. ÁLVAREZ, R. PLANAS, R. MORILLAS, \\ E. DOMÈNECH, E. CABRÉ, M. A. GASSULL
}

Servicio de Aparato Digestivo. Hospital Universitari Germans Trias i Pujol. Badalona. Barcelona

\author{
GASOMETRIC ALTERATIONS IN HOSPITALIZED CIRRHOTIC PA- \\ TIENTS
}

\section{RESUMEN}

Introducción y objetivos: Aunque se ha descrito la existencia de diversas alteraciones del intercambio gaseoso en la cirrosis, existen pocos estudios que las hayan estudiado de forma prospectiva. El objetivo de este trabajo fue conocer la frecuencia y gravedad de dichas alteraciones en los pacientes cirróticos hospitalizados, correlacionándolas con el grado de disfunción hepática.

Pacientes y métodos: Se estudiaron 50 pacientes cirróticos consecutivos (41 varones) que requirieron ingreso hospitalario por descompensación de su hepatopatia (ascitis, encefalopatía hepática, hepatitis alcohólica y hemorragia digestiva alta), y que no presentaban procesos pulmonares ni cardiacos agudos o crónicos que pudiesen producir hipoxemia. Los pacientes fueron agrupados según su estadio de Child-Pugh $(\mathrm{A}, \mathrm{n}=13 ; \mathrm{B}, \mathrm{n}=21 ; \mathrm{C}, \mathrm{n}=16)$. En siete pacientes se constató la presencia de una hepatitis alcohólica sobreañadida grave (HAAG). En todos ellos se realizó una gasometría arterial basal antes de ser dados de alta, y se efectuó un ecocardiograma transtorácico con contraste en caso de sospecha de síndrome hepatopulmonar (SHP).

Resultados: Se observó una discreta hipoxemia global $(80,9 \mathrm{mmHg})$ sin diferencias según el grado de Child-Pugh. La hipocapnia fue significativamente más marcada en los pacientes con estadio Child $\mathrm{C}$ que en aquellos con estadios A y B $(31,2 \pm 3,1$ frente a $38,1 \pm 4,3$ y $36,3 \pm 5$ mmHg; $\mathrm{p}<0,05)$, respectivamente. En cambio, los pacientes cirróticos con HAAG presentaron un hipocapnia significativamente menor que aquellos otros sin HAAG $(31,2 \pm 3,1$ frente a $36,3 \pm 5 \mathrm{mmHg} ; \mathrm{p}<0,05)$. En el análisis multivariante, las variables con valor pronóstico independiente para la presencia de hipocapnia fueron la protrombina, la albúmina y el sodio plasmáticos. Se constató la presencia de SHP en 8 pacientes $(16 \%)$.

Conclusiones: La alteración gasométrica más frecuentes de la cirrosis es la alteración del gradiente alvéolo-arterial de oxígeno, que se acentúa conforme empeora la función hepática. La hipocapnia, aunque su patogenia no es bien conocida, podría constituir una mecanismo compensador de la hipoxemia o bien ser el resultado de la activación de los centros respiratorios centrales por sustancias no aclaradas en el hígado.

PALABRAS CLAVE: Síndrome hepatopulmonar. Hipocapnia. Cirrosis. Hepatitis alcohólica.

\begin{abstract}
Background and objectives: Gas exchange alterations have been described in cirrhotic patients; but by the moment, a few prospective studies have focused in them. The aim of this study was to describe the frequency and severity of gasometric alterations in hospitalized cirrhotic patients, a their correlationwith hepatocellular disfunction.

Patients and methods: 50 consecutive cirrhotic patients (41 males) admited for liver decompensation (ascites, liver encephalopathy, alcoholic hepatitis and upper gastrointestinal bleeding) without acute or chronic cardiopulmonary disfunction were included in the study. Patients were classificated according with Child-Pugh score $(A, n=13 ; B, n=$ 21; $C, n=16)$. Severe alcoholic hepatitis (SAH) was confirmed in 7 patients. Arterial gasometry was performed in all patients before discharge. Contrast echocardiography was performed in any case of suspicion of hepatopulmonary syndrome (HPS).

Results: Light hypoxemia was observed $(80.9 \mathrm{mmHg})$, without differences with Child-Pugh. Hypocapnia was significantly more evident in Child $C$ than in $A$ and $B(31.2 \pm 3.1$ vs. $38.1 \pm 4.3$ y $36.3 \pm 5 \mathrm{mmHg} ; p<$ $0,05)$, respectively. Cirrhotic patients with SAH showed a significantly higher hypocapnia by comparison with others $(31.2 \pm 3.1$ vs. a $36.3 \pm 5$ $\mathrm{mmHg} ; \mathrm{p}<0.05)$. In multivariate analysis, independent prognostic variables for hypocapnica were plasmatic levels of protrombin time. albumin and sodium. HPS was confirmed in 8 patients (16\%).

Conclusions: The most prevalent gas exchange abnormality in cirrhosis was the alteration of alveolar-arterial oxygen tension gradient directly correlated with hepatocellur disfunction. Hypocapnia could be a compensatory mechanism or the result of the activation of central respiratory centres by non-depurated substances by the liver.
\end{abstract}

KEY WORDS: Hepatopulmonary syndrome. Hypocapnia. Cirrhosis Alcoholic hepatitis.

Lorenzo-Zúñiga V, Álvarez MA, Planas R, Morillas R, Doménech E, Cabré E, Gassull MA. Alteraciones gasométricas en pacientes cirróticos hospitalizados. An Med Interna (Madrid) 2005; 22: 209-212.

Trabajo aceptado: 10 de enero de 2005

Correspondencia: Vicente Lorenzo-Zúñiga García. Servicio de Aparato Digestivo. Hospital Universitari Germans Trias i Pujol. Ctra. del Canyet, s/n. 08916 Badalona. Barcelona. e-mail: vlorenzo@ns.hugtip.scs.es. 


\section{INTRODUCCIÓN}

Las alteraciones del intercambio gaseoso relacionadas con la cirrosis fueron descritas por primera vez por Flückiger en 1884 (1), aunque fue Sheila Sherlock quien inició su estudio en profundidad (2). En la hepatopatía crónica son varios los procesos que pueden ocasionar dichas alteraciones; como la ascitis, el derrame pleural, la hepatomegalia masiva, la debilidad de los músculos respiratorios, y especialmente el síndrome hepatopulmonar (SHP). El SHP define la presencia de hipoxemia arterial en pacientes afectos de hepatopatía crónica, en ausencia de enfermedad cardiaca o pulmonar intrínseca, asociada a dilataciones vasculares intrapulmonares. El SHP constituye una entidad clínica de prevalencia incierta, diagnóstico y tratamiento complejos y patogenia desconocida (3-5).

La hipoxia es un hecho frecuente y conocido en los pacientes que presentan una cirrosis $(6,7)$, que puede ser atribuida a la presencia de ascitis y derrame pleural. Sin embargo, en el SHP, la hipoxia es secundaria a los shunts intrapulmonares.

El objetivo del presente trabajo fue evaluar prospectivamente la frecuencia y gravedad de las alteraciones del intercambio gaseoso en los pacientes cirróticos hospitalizados, así como correlacionar dichas alteraciones con el grado de disfunción hepática.

\section{PACIENTES Y MÉTODOS}

Estudio clínico prospectivo y descriptivo de la prevalencia de las alteraciones gasométricas en pacientes cirróticos consecutivos que requirieron ingreso hospitalario por descompensación de su hepatopatía (ascitis, encefalopatía hepática, hepatitis alcohólica, hemorragia digestiva alta) entre enero y junio de 2002. Se excluyeron los pacientes que presentaban procesos pulmonares y cardiacos agudos o crónicos que pudiesen producir hipoxemia (antecedentes de asma bronquial o broncopatía crónica, incluyendo fumadores activos; presencia de hidrotorax hepático, ascitis a tensión y clínica de insuficiencia cardíaca). La cirrosis se diagnosticó de forma inequívoca por criterios clínicos, analíticos, ecográficos y endoscópicos.

A los pacientes que reunían criterios de inclusión se les informó de las características y objetivos del estudio y se obtuvo un consentimiento informado siguiendo las normas éticas de la declaración de Helsinki, que había sido previamente aprobado por el comité ético de nuestro centro. En los pacientes encefalopáticos el consentimiento se obtuvo a través su representante legal. Se procedió a realizar una una exploración física cardiopulmonar y una radiografía de tórax para confirmar la ausencia de patología cardiopulmonar, así como una gasometría arterial (GA) por punción percutánea de la arterial radical sin anestesia local en el lugar de punción, estando el paciente en decúbito supino respirando aire ambiente. La GA permitió determinar la presión parcial de oxígeno $\left(\mathrm{pO}_{2}\right)$ y de dióxido de carbono $\left(\mathrm{pCO}_{2}\right)$ mediante electrodos selectivos, utilizando un lector IL 16/40 pH/blood gas analyzer (Instrumentation laboratory SpA, Milan, Italia); cuyos valores normales son $\mathrm{pO}_{2}>80 \mathrm{mmHg}$ y $\mathrm{pCO}_{2}$ 35-45 $\mathrm{mmHg}$. El gradiente alveolo-arterial de oxígeno (AapO2) se calculó mediante la siguiente ecuación: $\mathrm{AapO}_{2}=[(\mathrm{PB}-47) \mathrm{x}$ (0.2093) - (1.15 x $\left.\left.\mathrm{pCO}_{2}\right)\right]-\mathrm{pO}_{2}$. (PB: presión barométrica), siendo los valores normales de $\mathrm{AapO}_{2} \leq 15 \mathrm{mmHg}$.

En todos los pacientes cirróticos se recogieron las siguientes variables clínicas y analíticas durante el ingreso hospitalario: edad, altura $(\mathrm{cm})$, peso $(\mathrm{Kg})$, presión arterial media ( $\mathrm{mmHg}$ ), frecuencia cardíaca (latidos/minuto), presencia de ascitis y encefalopatía hepática, tamaño de las varices esofágicas (ausencia, A, B, C, D), evidencia de arañas vasculares y telangiectasias, estadio de Child-Pugh, presencia de HAAG (hepatitis alcohólica asociada grave), definida por un índice de Maddrey superior a 32 (4,6 x [tiempo de protrombina en segundos: caso-control] + BilT); Hb (g/dl); AST (U/l); BilT (mg/dl); FA (U/l); tiempo de protrombina (\%); albúmina (g/l); Creatinina (mg/dl); $\mathrm{Na}(\mathrm{mmol} / \mathrm{l})$.

En los pacientes en los que se sospechó la presencia de un SHP $(8,9)$ se realizó un ecocardiograma transtorácica de contraste (ECTC) para confirmar la presencia de shunts intrapulmonares, utilizando suero salino al $0,9 \%$ como solución de contraste. En una vena periférica se inyectaron $10 \mathrm{~mL}$ de suero salino al $0,9 \%$ con $0,5 \%$ de aire mezclados tras hacerlos pasar 20 veces entre dos jeringuillas de $10 \mathrm{~mL}$ conectadas por una llave de tres pasos, de acuerdo con el método publicado (10). Se realizaron tres inyecciones en cada paciente; considerándose como resultado positivo la presencia del constraste en la aurícula izquierda 5-6 latidos cardíacos después de observarlas en la aurícula derecha.

\section{ANÁLISIS ESTADÍSTICO}

En el análisis descriptivo se utilizó el valor de mediaさdesviación estándar (DE) para expresar los resultados obtenidos. Para el análisis de las variables paramétricas se usó la diferencia de medias (prueba de la $t$ de Student) y para variables con más de dos categorías la prueba de ANOVA. En caso de variables no paramétricas empleamos la prueba U de MannWhitney o la $\mathrm{H}$ de Kruskal-Wallis, para dos o más variables, respectivamente. Para la comparación de proporciones se utilizó la prueba de la chi-cuadrado, utilizando un nivel de confianza del $95 \%$. Para la correlación entre dos variables de distribución normal se utilizó la prueba de Pearson y, como alternativa no paramétrica, se utilizó la de Spearman. Se realizó una regresión lineal múltiple para el estudio de asociaciones independientes, valorándose la significación estadística del modelo y cada una de las variables estudiadas, utilizando un nivel de signficación estadística del 95\%.

\section{RESULTADOS}

Se estudiaron 50 pacientes cirróticos (41 varones y 9 mujeres) con edades comprendidas entre los 48 y 66 años de edad (media 61,8 \pm 12,1 años). La etiología de la cirrosis, estadio de Child-Pugh y motivo de ingreso se exponen en la tabla I. Los parámetros clínicos y analíticos según el estadio de Child-Pugh se exponen en la tabla II.

La exploración física de los pacientes constató la presencia de arañas vasculares en 33 pacientes $(66 \%)$ y acropaquía en $7(14 \%)$. Las radiografías de tórax mostraban o bien un patrón normal o intersticial bilateral leve o moderado de predominio basal y aspecto moteado.

La $\mathrm{pO}_{2}$ media fue normal $(80,8 \pm 11,4)$, aunque inferior a la esperada según la edad de los pacientes (11). Veinte pacien- 
TABLA I

CARACTERÍSTICAS DEMOGRÁFICAS DE LOS PACIENTES, ETIOLOGÍA DE LA CIRROSIS Y MOTIVO DE INGRESO

\begin{tabular}{lc}
\hline Sexo masculino (\%) & 82 \\
Edad (años) & $61,8 \pm 12,1$ \\
Clasificación Child-Pugh (\%) & $13(26)$ \\
A & $21(42)$ \\
B & $16(32)$ \\
C & \\
Hepatitis alcohólica (\%) & $37(74)$ \\
No & $6(12)$ \\
IM<32 & $7(14)$ \\
IM>32 & \\
Etiología (\%) & \\
Alcohol & $23(46)$ \\
VHB/VHC & $20(40)$ \\
Alcohol + virus & $5(10)$ \\
Otras & $2(4)$ \\
Motivo de ingreso (\%) & \\
Ascitis & $28(56)$ \\
Encefalopatía hepática & $6(12)$ \\
HAA & $13(26)$ \\
Hemorragia digestiva & $3(6)$ \\
\hline
\end{tabular}

(IM: índice de Maddrey); (HAA: hepatitis aguda alcohólica).

\section{TABLA II}

PARÁMETROS CLÍNICOS Y ANALÍTICOS DE LOS PACIENTES SEGÚN EL ESTADIO DE CHILD-PUGH

\begin{tabular}{lcccc}
\hline & $A(n=13)$ & $B(n=21)$ & $C(n=16)$ & $p$ \\
\hline Edad & $63,9 \pm 10,5$ & $61,5 \pm 11,3$ & $62,2 \pm 13,5$ & n.s. \\
TAM (mmHg) & $101,7 \pm 15,5$ & $97,1 \pm 12,5$ & $93,1 \pm 8,1$ & n.s. \\
FC (L/min) & $74,1 \pm 13,6$ & $82,3 \pm 12,1$ & $76,4 \pm 10,9$ & n.s. \\
pO2 & $83,6 \pm 7,4$ & $78,5 \pm 13,4$ & $80,8 \pm 11,1$ & n.s. \\
pCO2 & $38,1 \pm 4,35$ & $35,6 \pm 5,1$ & $33,8 \pm 5,3$ & n.s. \\
AapO2 & $17,9 \pm 10,6$ & $26,7 \pm 14,9$ & $26,8 \pm 8,9$ & n.s. \\
Hb (g/L) & $12,6 \pm 2,1$ & $11,5 \pm 2,3$ & $11,4 \pm 1,8$ & n.s. \\
AST (UI/L) & $68,3 \pm 23,6$ & $79,2 \pm 53,1$ & $98,1 \pm 55,1$ & n.s. \\
BilT (mg/L) & $1,1 \pm 0,4$ & $2,9 \pm 3,6$ & $8,3 \pm 8,1$ & $<0,01$ \\
FA (UI/L) & $106,2 \pm 37,6$ & $106,1 \pm 47,1$ & $105,9 \pm 47,3$ & n.S. \\
Tprot (\%) & $75,1 \pm 13,6$ & $70,4 \pm 14,5$ & $48,4 \pm 8,3$ & $<0,001$ \\
Alb (g/L) & $35,5 \pm 5,6$ & $28,8 \pm 4,1$ & $24,1 \pm 1,9$ & $<0,001$ \\
Creat (mg/L) & $0,8 \pm 0,1$ & $0,8 \pm 0,3$ & $0,7 \pm 0,2$ & n.S. \\
Na (mmol/L) & $137,1 \pm 53,1$ & $135,2 \pm 28,1$ & $133,4 \pm 4,6$ & $<0,05$ \\
\hline
\end{tabular}

(TAM: tensión arterial media); (FC: frecuencia cardíaca); ( $\mathrm{Hb}$ : hemoglobina); (AST: aspartato aminotransferasa); (BilT: bilirrubina total); (FA: fosfatasa alcalina); (Tprot: tiempo de protrombina); (Alb: albúmina); (Creat: creatinina); (Na: sodio).

tes $(40 \%)$ presentaron hipoxemia leve $\left(\mathrm{pO}_{2}>60\right.$ y $<80$ $\mathrm{mmHg}$ ) y sólo dos pacientes $(4 \%)$ presentaron hipoxemia grave $\left(\mathrm{pO}_{2}<60 \mathrm{mmHg}\right)$, sin que ello se relacionar con el ChildPugh. En cambio, la presencia de acropaquía se correlacionó de forma estadísticamente significativa con la presencia de hipoxemia $(71 \pm 9,5$ frente a $82,4 \pm 10,9 \mathrm{mmHg}$; $\mathrm{p}<0,05)$, respectivamente.

Se detectó hipocapnia $\left(\mathrm{pCO}_{2}<35 \mathrm{mmHg}\right)$ en 23 pacientes (46\%). La existencia de hipocapnia se correlacionó estadísticamente con el estadio Child-Pugh C (31,2 $\pm 3,1$ frente a 38,1 $\pm 4,3$ y $36,3 \pm 5 \mathrm{mmHg}$, en los estadios A y B, respectivamente; $\mathrm{p}<0,05)$; y con la presencia de encefalopatía hepática $(31,8$ $\pm 4,3$ frente $36,1 \pm 5,11 \mathrm{mmHg} ; \mathrm{p}<0,05$ ).

La presencia de un $\mathrm{AapO}_{2}>15 \mathrm{mmHg}$ fue la alteración gasométrica más frecuente, detectándose en 40 pacientes (80\%). Este porcentaje se redujo al $62 \%$ cuando la $\mathrm{AapO}_{2}$ fue $>20 \mathrm{mmHg}$, siendo más evidente y estadísticamente significativa en los estadios B y C de Child $(26,7 \pm 3,2$ y $26,1 \pm 2,2$ $\mathrm{mmHg})$ que en el A $(17,9 \pm 2,9 \mathrm{mmHg} ; \mathrm{p}<0,05)$; así como en los pacientes que presentaban telangiectasias $(26,5 \pm 12,8$ frente 19,8 $\pm 12,5 \mathrm{mmHg}$; $\mathrm{p}<0,05$ ).

En 12 pacientes (24\%) se diagnosticó una HAA; siendo ésta grave (HAAG, índice de Maddrey > 32) en 7 pacientes (14\%). La presencia de una HAAG se asoció a una mayor alteración de la $\mathrm{pCO}_{2}$ y $\mathrm{AapO}_{2}$ en comparación con los pacientes sin HAA $(31,2 \pm 3,1$ frente a $36,3 \pm 5 \mathrm{mmHg})$ y $(30,5 \pm 6,1$ frente a $23 \pm 2 \mathrm{mmHg}$ ), respectivamente; $\mathrm{p}<0,05$ en ambas comparaciones. En el análisis multivariante las únicas variables con valor predictivo independiente de mayor disfunción respiratoria $\left(\mathrm{pCO}_{2}<35 \mathrm{mmHg}\right.$ y $\mathrm{AapO}_{2}>20 \mathrm{mmHg}$ ) fueron el tiempo de protrombina y los niveles plasmáticos de albúmina y sodio $(\mathrm{p}<0,05)$, tal y como se refleja en la tabla III.

TABLA III

\begin{tabular}{|c|c|c|c|}
\hline \multicolumn{4}{|c|}{ TABLA III } \\
\hline \multicolumn{4}{|c|}{$\begin{array}{l}\text { VALOR DE P EN EL ANÁLISIS UNIVARIANTE Y } \\
\text { MULTIVARIANTE }\end{array}$} \\
\hline & $\mathrm{O}_{2}(\mathrm{mmHg})$ & $\mathrm{AapO}_{2}(\mathrm{mmHg})$ & $\mathrm{pCO}_{2}(\mathrm{mmHg})$ \\
\hline Bilirrubina total (mg/L) & 0,850 & 0,878 & 0,550 \\
\hline Tiempo protrombina (\%) & 0,374 & $0,041^{*}$ & $0,040^{*}$ \\
\hline Albúmina (g/L) & 0,563 & $0,048^{*}$ & $0,041^{*}$ \\
\hline Creatinina (mg/L) & 0,868 & 0,757 & 0,406 \\
\hline Sodio plasmático (mmol/L) & ) 0,410 & 0,549 & $0,045^{*}$ \\
\hline
\end{tabular}

Se realizó una ecografía bidimensional de contraste en 8 pacientes $(16 \%)$, por sospecha de SHP $\left(\mathrm{pO}_{2}<70 \mathrm{mmHg}\right.$ y $\mathrm{AapO}_{2}>20 \mathrm{mmHg}$ ), que confirmaron la presencia de shunts vasculares intrapulmonares. La presencia de un SHP se correlacionó de forma estadísticamente significativa con la presencia de encefalopatía hepática $(\mathrm{p}<0,0001)$, acropaquía ( $\mathrm{p}<0,0001)$ y telangiectasias $(\mathrm{p}<0,05)$. En cambio, no se evidenció ninguna correlación entre la presencia de SHP y el tamaño de las varices esofágicas o la presencia de ascitis.

\section{DISCUSIÓN}

En la cirrosis se han constatado alteraciones del intercambio gaseoso que todavía no han sido aclaradas en su totalidad y cuya prevalencia no es del todo conocida. La prevalencia de la hipoxemia en pacientes cirróticos con hepatopatía compensada se situa entre el $30-60 \%(12,13)$, siendo a menudo asintomática y sólo ocasionalmente incapacitante $(6,7)$. En el pre- 
sente estudio, los pacientes cirróticos hospitalizados presentaron una prevalencia de hipoxemia leve del $40 \%$, similar a lo referido en la literatura; mientras que sólo en un $4 \%$ presentan una insuficiencia respitatoria.

Un aspecto interesante del presente estudio fue la observación de que la hipocapnia $\left(\mathrm{pCO}_{2}<35 \mathrm{mmHg}\right.$ ) y las alteraciones del $\mathrm{AapO}_{2}$ fueron estadísticamente significativos en los pacientes que presentaban una mayor disminución de la función hepatocelular (pacientes con estadios B y C de Child-Pugh) $(14,15)$ y en los que presentaban una HAAG (16). La HAAG produce una insuficiencia hepática grave y un estado circulatorio hiperdinámico que pueden contribuir al mayor empeoramiento gasométrico observado en estos pacientes. La hiperventilación es el mecanismo por el que estos pacientes presentan una cifras normales o casi normales de $\mathrm{pO}_{2}$ (17); es por ello que el $\mathrm{AapO}^{2}$ es el mejor parámetro para detectar las alteraciones del intercambio gaseoso en estos pacientes $(17,18)$. En nuestro estudio, las variables independientes predictoras de una mayor alteración del intercambio gaseoso que presentaron un mayor peso estadístico, y que por lo tanto mejor describen el deterioro de la función hepática, fueron el tiempo de protrombina y los niveles plasmático del sodio y albúmina.

\section{Bibliografía}

1. Flückiger M. Vorkomen von Trommelschlagel förmigen Fingerendphalanger ohne chronische Veränderungen an den Lunger oder am Herzen. Wied Med Wochenschr 1884; 34: 1457.

2. Sherlock S. The liver-lung interface. Semin Respir Med 1988; 9: 24753.

3. Krowka MJ, Cortese DA. Hepatopulmonary syndrome: Current concepts in diagnostic and therapeutic considerations. Chest 1994; 105: 1528-37.

4. Rodríguez-Roisin R, Agustí AGN, Roca J. The hepatopulmonary syndrome: New game, old complexities. Thorax 1992; 47: 897-902.

5. Castro M, Krowka MJ. Hepatopulmonary syndrome: A pulmonary vascular complication of liver disease. Clin Chest Med 1996; 17: 35-48.

6. Edell ES, Cortese DA, Krowka MJ, Rehder K. Severe hypoxemia and liver disease. Am Rev Respir Dis 1989; 140: 1631-5.

7. Colle I, Langlet P, Barriere E, Heller J, Rassiat E, Condat B, et al. Evolution of hypoxemia in patients with severe cirrhosis. J Gastroenterol Hepatol 2002; 17: 1106-9

8. Henemann HO, Emirgil C, Mijnssen JP. Hyperventilation and arterial hypoxemia in cirrhosis of the liver. Am J Med 1960; 28: 239-46.

9. Gupta D, Vijaya DR, Gupta R, Dhiman RK, Bhargaca M, Verma J, et al. Prevalence of hepatopulmonary syndrome in cirrhosis and extrahepatic portal venous obstruction. Am J Gastroenterol 2001; 96: 33953399.

10. Hind CR, Wong CM. Detection of pulmonary arteriovenous fistulae in patients with cirrhosis by contrast 2D echocardiography. Gut 1981;21: $1042-5$.

11. RO Crapo, RL Jensen, M Hegewald, DP Tashin, Arterial Blood Gas Reference Values for Sea Level and an Altitude of 1,400 Meters. Am J Respir Crit Care Med 1999; 160: 1525-1531

12. Kennedy TC, Knudson RJ. Exercise-agravate hypoxemia and orthodeoxia in cirrhosis. Chest 1977; 72: 305-9.

13. Moller S, Hillings J, Chirstiensen E. Arterial hypoxaemia in cirrhosis:
Por otra parte, la prevalencia del SHP mediante ecocardiograma con contraste transtorácico detectada en nuestro estudio (16\%) es similar a la descrita en estudios previos $(14,19-24)$, teniendo en cuenta de que en nuestro estudio no se realizó un cribado de SHP en todos los pacientes. La presencia del SHP fue significativamente superior en aquellos pacientes con presencia de encefalopatía hepática, acropaquias y telangiectasias, confirmado la correlación estadística entre estas variables y las alteraciones del intercambio gaseoso, siendo más evidentes a mayor deterioro de la función hepática. En cambio, y al igual que el estudio de Krowka y cols. (14), la existencia de un SHP no se correlacionó con el tamaño de las varices esofágicas ni con la presencia de ascitis. En conclusión, la alteración gasométrica más frecuente en este grupo de pacientes crirróticos hospitalizados es la alteración del gradiente alvéolo-arterial de oxígeno, seguido de la hipocapnia, que se acentúan conforme empeora la función hepática. Aunque la patogenia de la hipocapnia no es bien conocida, podría constituir una mecanismo compensador de la hipoxemia o bien ser el resultado de la activación de los centros respiratorios centrales por sustancias no aclaradas en el hígado. fact or fiction? Gut $1998 ; 42: 868-874$

14. Krowka MJ, Dickson ER, Cortese DA. Hepatopulmonary syndrome: clinical observations and lack of therapeutic response to somatostatin analogue. Chest 1993; 104: 515-521

15. Aller R, Moya JL, Moreira V, Boixeda D, Picher J, García-Rull S et al. Etiology and frequency of gas exchange abnormalities in cirrhosis. Rev Esp Enferm Dig 1999; 91: 564-568.

16. Robrerol J, Corradi P, Chahuan M. Hepatopulmonary syndrome in decompensated cirrhotic patients. Rev Med Chil 1995; 123: 975-981.

17. Lange PA, Stoller JK. The hepatopulmonary syndrome. Ann Intern Med 1995; 122: 521-9.

18. Bashour FA, Cochran P. Alveolar-arterial oxygen tension gradients in cirrhosis of the liver. Futher evidence of existing pulmonary arteriovenous shunting. Am Heart J 1966; 71: 734-40.

19. Fahy JV, Kerr KM, Lake JR, Gold WM. Pulmonary function before and after liver transplantation (Abstr). Am Rev Respir Dis 1992; 143: A303.

20. Abrams G, Jaffe C, Hoffer P. Diagnostic utility of contrast echocardiography and lung perfusion scan in patients with hepatopulmonary syndrome. Gastroenterol 1995; 109: 1283-1288.

21. Vedrinne JM, Duperret S, Bizollon T. Comparison of transesophageal and transthoracic contrast echocardiography for detection of an intrapulmonary shunt in the liver disease. Chest 1993; 111: 12361239.

22. Herve P, Lebrec D, Brenot F. Pulmonary vascular disorders in portal hypertension. Eur Respir J (deb) 1998; 11: 1153-66.

23. Mimidis KP, Karatza C, Spiropoulos KY. Prevalence of intrapulmonary vasculars dilatations in normoxaemic patients with early liver cirrhosis. Scan J Gastroenterol 1997; 33: 988-92.

24. Schenk P, Fuhrmann V, Madl C, Funck G, Lehr S, Kandel O, et al. Hepatopulmonary syndrome: prevalence and predictive value of various cut offs for arterial oxygenation and their clinical consequences. Gut 2002; 51: 853-859. 